\title{
Norois
}

Environnement, aménagement, société

197 | 2005/4

Varia

\section{La question environnementale en géographie au Brésil : une approche épistémologique}

The environmental question in geography in Brazil: an epistemological approach

\section{Francisco Mendonça}

\section{(2) OpenEdition}

\section{Journals}

\section{Édition électronique}

URL : http://journals.openedition.org/norois/311

DOI : 10.4000/norois.311

ISBN : 978-2-7535-1545-1

ISSN : $1760-8546$

Éditeur

Presses universitaires de Rennes

Édition imprimée

Date de publication : 1 décembre 2005

Pagination : 55-67

ISBN : 978-2-7535-0238-3

ISSN : 0029-182X

\section{Référence électronique}

Francisco Mendonça, «La question environnementale en géographie au Brésil : une approche épistémologique », Norois [En ligne], 197 | 2005/4, mis en ligne le 15 décembre 2008, consulté le 19 avril 2019. URL : http://journals.openedition.org/norois/311 ; DOI : 10.4000/norois.311

Ce document a été généré automatiquement le 19 avril 2019.

(c) Tous droits réservés 


\section{La question environnementale en géographie au Brésil : une approche épistémologique}

The environmental question in geography in Brazil: an epistemological approach

\section{Francisco Mendonça}

\section{NOTE DE L'ÉDITEUR}

Traduit du portugais par Nathalie Dessartre

« La crise environnementale n'est pas une crise écologique, mais une crise de la raison. Les problèmes environnementaux sont, fondamentalement, des problèmes de savoir. D'où peuvent être dérivées de fortes implications pour toute politique environnementale - qui se doit de passer par une politique du savoir -, et aussi pour

l'éducation. Appréhender la complexité environnementale ne constitue pas un problème d'apprentissage du milieu, mais aussi de compréhension du savoir sur ce milieu. »

(Leff, 2001)

Toile de fond d'une profonde crise de civilisation (Hobsbawn, 1995) liée à de grands changements, la plus grave de l'humanité (Monteiro, 1988), la fin du xxe siècle et le début du xxI ${ }^{e}$ siècle défient la société en général de trouver de nouvelles voies pour construire le présent et le futur. Aux intellectuels et aux hommes de science, elle demande globalement de repenser l'ontologie et l'épistémologie de la science en remettant en question les paradigmes qui servent d'appui à la production du savoir de la modernité. Aux géographes, en particulier, la société impose une profonde remise en question à 
propos du statut de la géographie contemporaine face aux nouvelles dimensions de l'espace et aux graves problèmes sociaux qui prennent forme sur la surface terrestre.

C'est dans la tentative de caractériser la spécificité de la science géographique au sein de crises et de changements contemporains, «[...] - un monde confus, de géographies confuses $-[. .$.$] » d'après C. A. F. Monteiro (1988), et parallèlement de contribuer à$ l'approfondissement du débat sur l'épistémologie de la géographie et de montrer des chemins pour son développement que ce texte a été élaboré.

Le choix du thème central de la présente analyse - géographie et environnement procède tant d'une réflexion construite au long des vingt dernières années à partir de lectures, de débats et d'expérience professionnelle, que de préoccupations consécutives à l'investissement croissant de nombreux géographes dans des activités techniques et didactiques liées aux problèmes environnementaux actuels. Différentes réflexions et pratiques observées ont mis en évidence la nécessité d'un exercice théorique en vue d'identifier et de distinguer l'histoire et les principales caractéristiques d'un segment qui, au sein de la géographie, semble actuellement se constituer en un courant de pensée de cette même science.

Ce thème est fondamental actuellement, même s'il est certain que l'origine de la crise écologique remonte à bien avant la modernité comme l'a si bien démontré L. White Jr (1968). Il prend toute son importance dans la mesure où l'on conçoit qu'» un des aspects le plus remarquable dans la présente crise historique est celui qui est né de l'état de carence dans lequel le développement industriel et technologique, orienté par le droit de veto que l'homme s'est attribué sur la nature, a produit dans la qualité environnementale et dans les ressources naturelles [...]» (Monteiro, 1988).

Parler de la problématique environnementale et de son approche en géographie implique d'aborder une des principales discussions qui ont marqué le dernier quart de siècle (et même plus !) des débats des géographes, soit la dichotomie ou la dualité entre géographie physique et géographie humaine, tant au Brésil (Mendonça, 1989, 1993, 1998) qu'en France (Trystram, 1994; Bravard, 1999). Il est alors nécessaire de préciser que dans ce texte une telle approche est associée à la perspective de la dualité du savoir géographique, car nous croyons que c'est là sa plus grande richesse (Mendonça, 1998). Croyance qui met en évidence tant la nature «[...] essentiellement transdisciplinaire [... ]», et interdisciplinaire de la géographie, que le fait que celle-ci soit « [...] un des derniers locus du naturalisme dans les sciences humaines » (Moraes, 1994) et de l'humanisme dans les sciences naturelles. Ce point de vue prend en considération la conviction selon laquelle l'approche géographique de l'environnement transcende la vieille discussion de la dichotomie géographie physique versus géographie humaine, puisqu'elle conçoit l'unité du savoir géographique comme consécutive de l'interaction entre les différents éléments et facteurs qui composent son objet d'étude.

\title{
Géographie, environnement et environnementalisme
}

\author{
« La géographie est un savoir, un savoir difficile \\ parce qu'intégrateur du vertical et de l'horizontal, \\ du naturel et du social, de l'aléatoire et du \\ volontaire, de l'actuel et de l'historique et sur la \\ seule interface dont dispose l'humanité. » \\ (P. et G. Pinchemel dans Trystram, 1994)
}


Le débat sur l'environnement a fortement marqué l'histoire de la société humaine du dernier quart du $\mathrm{xx}^{\mathrm{e}}$ siècle et s'est intégralement propagé dans le savoir géographique.

7 Le $\mathrm{xx}^{\mathrm{e}}$ siècle a assisté à la lente transformation de la connotation des termes environnement et environnementalisme parce que, jusqu'aux années 1950, les discussions relatives à cette thématique avaient encore une conception majoritairement naturaliste et scientifique. Ainsi, on peut observer l'évolution du concept d'environnement dans les mots suivants de A. Bailly et R. Ferras (1997) : «En 1917, l'environnement, c'est pour une plante "the resultant of all the external factors acting upon it". En 1944, pour un organisme "the sum total effective factors to which an organism responds". En 1964, Harant et Jarry proposent: "Ensemble des facteurs biotiques (vivants) ou abiotiques (physico-chimique) de l'habitat." En 1971 selon Ternisien: "Ensemble, à un moment donné, des agents physiques, chimiques et biologiques et des facteurs sociaux susceptibles d'avoir un effet direct ou indirect, immédiat ou à terme, sur les êtres vivants et les activités humaines." Et voilà le mot à la mode, en proie à l'inflation journalistique [...]. »

Dans l'évolution du concept d'environnement (environment), on remarque que l'insertion des activités humaines est plus contemporaine, cependant, le fait que l'homme socialement organisé semble se constituer plus en facteur qu'en élément de l'environnement conduit à penser que le concept d'environnement est toujours fortement lié à une conception naturaliste. En général, et si l'on observe le sens commun, tout comme le débat intra et extra académique, on a l'impression qu'aborder l'environnement signifie parler automatiquement de la nature, comme si dans ce champ il y avait une hiérarchisation des éléments où ceux qui se réfèrent au cadre naturel sont (hiérarchiquement) en position supérieure.

9 Cependant, il est notoire que l'emploi du terme portugais "meio-ambiente " (environnement) est actuellement devenu embarrassant pour les spécialistes lusophones en environnement parce que, comme l'a montré C. W. Porto Gonçalves (1989), le fait que «milieu » signifie aussi moitié, partie, portion, sous-tend l'idée d'une approche partielle des problèmes environnementaux. Même si cette lecture critique présente une cohérence étymologique considérable, il est cependant regrettable que les géographes n'aient pas fait plus d'efforts pour expliquer la spécificité et l'importance du terme "meio», du moins son sens scientifique, parce que l'emploi de ce dernier dans un contexte environnemental correspond actuellement à une dérivation, voire à une appropriation générale du concept de milieu géographique. Il faut aussi faire attention au fait que de nombreux géographes considèrent le terme «ambiente» (environnement) comme «presque synonyme » du terme géographie, au point de concevoir l'emploi d'expressions telles que « géographie environnementale » comme réductionniste.

Ce dernier concept (milieu géographique), très bien utilisé par Albert Demangeon et par Élisée Reclus au début du siècle dernier, a permis d'ouvrir et de faire avancer la compréhension holistique des différents espaces géographiques de la planète dans une perspective environnementale globalisante. Ainsi, bénéficiaire d'un héritage d'une telle importance, la conception géographique de l'environnement permet à l'approche environnementale de suivre une nouvelle voie, comme l'a affirmé Y. Veyret (1999) : «En fait pour un géographe, la notion d'environnement ne recouvre pas seulement la nature, encore moins la faune et la flore seules. Ce terme désigne les relations d'interdépendance 
qui existent entre l'homme, les sociétés et les composants physiques, chimiques, biotiques du milieu en intégrant aussi ses aspects économiques, sociaux et culturels. »

Bien que devenu plus large, le terme «meio ambiente»- ou "ambiente» (qui eux, peuvent être envisagés comme des synonymes), semble ne pas arriver à se détacher d'une genèse et d'une histoire que des principes naturalistes ont profondément marqués. Ceci conduit à croire qu'était née une conception culturelle de l'environnement dans laquelle la société n'était pas considérée comme une composante, mais comme un acteur intervenant sur le milieu. Insérer au sein de l'approche environnementale la perspective humaine - par conséquent sociale, économique, politique et culturelle - semble être un défi pour toute une génération d'intellectuels, de scientifiques et d'environnementalistes qui participent à de telles discussions dans le présent et dans le futur.

Ainsi, face à un défi si important, on observe, de nos jours, une forte tendance à utiliser au sens large le terme socio environnemental, parce qu'il est devenu difficile et insuffisant de parler d'environnement du seul point de vue naturel lorsque l'on pense à la problématique interaction société-nature présente et future, surtout pour les pays dont le développement est complexe (Mendonça, 1993).

Le terme "socio " apparaît alors associé au terme "environnemental» de manière à mettre en relief le fait que la prise en compte de la société en tant que sujet, élément, partie fondamentale des processus liés à la problématique environnementale contemporaine est nécessaire.

La réalisation de la Conférence des Nations Unies pour le Développement et l'Environnement, aussi appelée Rio-ECO/92, marque principalement cette évolution conceptuelle. Les débats qui ont vu le jour lors de cette manifestation ont provoqué, entre autres, des changements des conceptions liées à l'environnement; ils ont engendré de nouveaux éléments qui ont transformé la conception des problèmes environnementaux (Mendonça, 1993). L'importance adéquate donnée à la dimension sociale de ces problèmes a rendu possible l'emploi de la terminologie socio-environnementale, qui est d'une importance fondamentale en géographie.

Cependant, le débat portant sur l'étymologie liée à la problématique environnementale ne prend pas fin avec l'adoption d'une ou d'une autre terminologie, comme nous en a averti A. C. R. Moraes (1994), à propos des fondements épistémologiques pour l'étude de l'environnement, lorsqu'il a souligné qu'un des principaux problèmes liés à ce champ d'études venait de sa diversité conceptuelle et de langage. D'après l'auteur: «[...] Le terme écologie, par exemple - et personne ne va nier qu'il est au centre des discussions -, apparait dans certains contextes discursifs comme un objet; alors que, dans d'autres contextes, il apparait comme méthode; ou dans d'autres encore, comme science, et même dans certains, comme question politique. Nous avons alors un terme qui varie beaucoup, en fonction du contexte discursif où il est utilisé. »

La situation du terme environnement est similaire, marqué de conceptions et de concepts pluriels, et présent tant dans des acceptions scientifiques, politiques et culturelles, que politico-gouvernementales, dans des mouvements sociaux en général, entre autres. Mais sur un point il semble y avoir concordance: les termes environnement et environnementalisme employés de nos jours sont liés à des conceptions complètement différentes de celles de la fin du $\mathrm{XIX}^{\mathrm{e}}$ et du début $\mathrm{du} \mathrm{xx}^{\mathrm{e}}$ siècle. Si à l'époque ils étaient associés directement à l'étude de la nature de la planète, aujourd'hui ils sont plus liés aux 
problèmes graves nés de l'interaction entre la société et la nature, à la relation hommemilieu, homme-nature, physique-humain, homme-homme, etc.

Pourtant, le contexte récent où naissent les grandes discussions et défis environnementaux semble ne plus permettre à l'homme de créer des situations où le scientifique, l'intellectuel, le technicien, le professionnel et le citoyen soient des éléments déconnectés. Pour ceux qui vivent dans le monde académique et institutionnel de la recherche un quotidien concerné en permanence par les problèmes environnementaux, il est, très souvent, difficile de séparer l'écologique de l'écologisme, l'environnement de l'environnementalisme. De leur côté, ceux qui sont plongés dans les mouvements environnementaux demandent sans cesse une base scientifique pour plaider leurs causes.

Dans le cas particulier de la géographie en tant que science, on observe une très étroite relation entre elle et le traitement de l'environnement et de la problématique de l'environnement, qui est une des caractéristiques les plus explicites de la géographie depuis le XviIIe siècle où elle est devenue science moderne (Moraes, 1994; Mendonça, 1989, 1993).

\section{Géographie écologique, géographie environnementale, géographie socio-environnementale : construction historique et particularités}

19 Analysée dans le champ de la pensée géographique moderne, l'approche environnementale peut être conçue à partir de deux grands moments, d'après F. Mendonça (1993). Le premier, dans lequel l'environnement est pris comme synonyme de nature (environnementalisme = naturalisme), a prévalu depuis la structuration de la science géographique jusqu'à la moitié du $\mathrm{xx}^{\mathrm{e}}$ siècle, bien qu'il soit encore possible de l'observer comme prise de position philosophique face au monde de la part de nombreux scientifiques et intellectuels, géographes inclus. À cette première période, on pourrait aussi associer les approches naturaliste et fonctionnaliste de la géographie conçues par $\mathrm{P}$. Claval (2001), et même le technicisme, la deuxième prise de position que A. C. R. Moraes (1994) identifie chez les scientifiques actuels face à la problématique environnementale.

20 C'est dans cette deuxième étape que l'on remarque le saut effectué par les géographes lorsqu'ils rompent avec la caractéristique majoritairement descriptive-analytique de l'environnement naturel, même si elle existe toujours (Mendonça, 1993). Cette démarche se caractérise par l'approche, dans la perspective de l'interaction société-nature, de manière détaillée et consciente, de l'analyse et de propositions d'interventions en vue de restaurer la dégradation de l'environnement et d'améliorer la qualité de vie de l'homme.

21 Dans cette deuxième phase de l'environnementalisme géographique, on remarque une différence significative entre le courant environnementaliste et d'autres courants contemporains de la pensée géographique, en particulier celui de la géographie critique brésilienne. Si pour cette dernière, l'échec du socialisme réel et la remise en question de la perspective marxiste comme prisme nécessaire à la lecture du réel s'amoindrissent, pour le courant environnemental l'impact n'a pas été si profond, car en fin de compte il est devenu plus clair que la recherche de solutions aux problèmes socioenvironnementaux de la planète doit être au-dessus de toutes idéologies, même si toutes peuvent se l'approprier. 

$\mathrm{y}$ ait eu une ressemblance parfaite entre la géographie critique et la géographie environnementale, ni même qu'au sein de la géographie critique brésilienne, le traitement de la question environnementale ait été ample et satisfaisant. Pour beaucoup de géographes, d'un pays ou de l'autre, la perspective environnementale ne semble pas avoir été suffisamment significative pour donner naissance à un nouveau courant de la pensée géographique, comme le dénote la constatation suivante de A. Bailly et R. Ferras (1997) : « Depuis 1975, la nouvelle géographie s'approfondit en de multiples courants qui se renforcent : géographie critique, géographie des représentations, géographie politique, géographie théorique, géographie culturelle, géographie humaniste [...]»; on remarque ici l'absence de la géographie environnementale. Il en est de même dans l'entendement d'une bonne partie des géographes qui sont plus proches des aspects humains de la géographie.

Un tel mépris, de la part de certains géographes - attitude assimilable à une prise de position technocentriste (Foladori, 1999) face à la problématique environnementale actuelle -, peut être dû à divers facteurs, entre lesquels on peut citer :

- L'option en faveur de l'entendement de la géographie comme une science éminemment sociale pour laquelle le support physico-naturel (même modifié) semble être secondaire ou sans importance tant du point de vue de la structuration spatiale de la société que de celui de l'influence de la nature sur elle ou vice versa ;

- La prise de distance volontaire par rapport à la problématique environnementale de la planète, ce qui peut révéler le fait de croire que la technologie à l'origine des problèmes environnementaux, leur trouvera aussi une solution et que, par conséquent, ceux-ci ne constituent pas des objets de premier ordre pour l'intérêt géographique ;

- La méconnaissance et l'incapacité de comprendre la dynamique de la nature et son importance dans la constitution de l'espace, du territoire et de la société.

À ce propos, et sans esprit polémique mais dans le même sens que les arguments de D. Massey (1999), il faut reconnaître le considérable effort de nombreux géographes physiciens en matière de compréhension et d'insertion des processus sociaux dans leur interaction avec la nature des paysages et dans les problèmes environnementaux, effort qui est encore modeste chez les géographes humains dans leur appréhension de la nature dans l'étude de la société.

La prise de position idéologique explicite du courant critique au Brésil et son lien avec la méthode marxiste qui a servi de base à l'élaboration de l'étude de l'espace et de l'environnement n'ont permis que des traitements partiels de ces derniers, même si une bonne quantité de travaux d'excellente qualité ont été élaborés dans cette perspective. Ce qui s'est passé au Brésil, dans les années soixante-dix et quatre-vingt, quand parmi les militants du courant de la géographie critique se trouvaient des géographes physiciens, semble légèrement rappeler ce qui s'est passé en France dans les années cinquante et soixante. Dans ce pays, un groupe de géographes physiciens (Jean Dresch, Jean Tricard, etc.) militait au parti communiste et/ou dans des partis de gauche et, au même moment, étudiait des phénomènes liés au cadre naturel de la planète ; au Brésil, près de vingt ans après, on peut citer Aziz Ab'saber, Claudio de Mauro, Dirce Suertegaray, Wanda Sales, Francisco Mendonça, etc.

Le militantisme de gauche a beaucoup appris à ces géographes sur les nouvelles perspectives d'analyse de l'espace et de l'environnement, considérant les progrès ainsi 
introduits dans l'étude du paysage par l'expérience politique. En ce qui concerne la géographie, produite par eux, on peut affirmer qu'elle a été osée et innovatrice lorsqu'elle a dépassé les limites caractéristiques de la géographie classique ou traditionnelle et lorsqu'elle a inséré la perspective analytique et critique générale dans les modèles en vigueur dans la société et dans la science naturelle; par contre ces mêmes géographes se sont révélés incapables d'insérer le marxisme, en tant que méthode, dans la dynamique évolutive des éléments de la nature dans le devenir des paysages.

On ne peut, par conséquent, admettre qu'une telle production géographique fasse totalement partie du courant de la géographie physique, ou qu'elle ait été produite cent pour cent conforme aux principes du courant critique brésilien, parce que la science que ces géographes ont produite, revêt une posture critique de grande importance pour la production de savoir, mais pas une application de la perspective marxiste dans l'analyse détaillée de l'évolution du paysage et des problèmes environnementaux. La méthode de recherche scientifique utilisée par ces géographes explique les différences fondamentales qui existent entre la prise de position critique générale et celle de la critique marxiste. B. M. Soares Pontes (1999), à propos de la relation nature/société du point de vue marxiste, présente des arguments très importants pour comprendre cette distinction lorsqu'il considère que "l'histoire de la nature précéderait l'histoire de l'humanité, mais à partir du moment où celle-ci aurait atteint un niveau élevé de développement technologique et agirait avec une efficacité de plus en plus grande, afin de modifier la nature, l'histoire naturelle serait subordonnée à l'histoire sociale et ferait partie intégrante de celle-ci. Le grand souci de cette ligne interprétative n'est évidemment pas l'étude de la nature en soi, mais le fondement du socialisme comme suite logique du capitalisme, comme "étape " historique postérieure et plus avancée, dans une interprétation évolutionniste/ transformiste.»

La nature ne doit pas du tout être envisagée à partir de méthodes spécifiques aux études de la société, de même que la société ne doit pas l'être à partir de méthodes qui relèvent des sciences naturelles, bien que la perspective de la problématique environnementale parte d'une optique sociale. À ce propos, et pour enrichir cette discussion, il est intéressant et éclairant d'observer l'interprétation de H. Leff (2001), pour qui «[...] à partir du moment où la nature devient, dans un processus général, un objet scientifique l'évolution biologique, la dynamique des écosystèmes -, ces objets biologiques doivent inclurent les effets des relations sociales de production qui les affectent. Et ces effets doivent être considérés dans leurs déterminations socio-historiques spécifiques, non dans la réduction du social et de l'histoire en processus naturels ou écologiques. À partir du moment où la nature se transforme en objet de processus de travail, le naturel est absorbé par le matérialisme historique. Ceci ne nie pas les lois biologiques des organismes qui font partie du processus, y compris l'homme et sa force de travail ; mais le naturel se transforme en biologique surdéterminé par l'histoire. Ni la ressource naturelle, ni la force de travail se réfèrent au métabolisme biologique ou à la dépense énergétique des organismes vivants. La ressource naturelle et la force de travail ne sont pas des objets naturels qui existent indépendamment du social, mais sont déjà le biologique déterminé par les conditions de production et de reproduction d'une structure sociale donnée. »

Cette dernière interprétation, bien qu'explicitement guidée par une vision du monde et de l'environnement propre à la perspective marxiste, ne cesse pour autant d'attirer l'attention sur le fait qu'il faille distinguer les « lois naturelles » (dynamique de la nature) et les processus sociaux (dynamique de la société) concernés par la genèse de la 
problématique environnementale contemporaine. La géographie socio-environnementale ne doit pas renoncer à cette perspective.

À propos du courant écologique et de sa dérive au sein de la géographie, ce qui aurait donné lieu à une "géographie écologique ", M. C. Andrade (1987) considère comme une de ses références importantes la publication de la proposition d'une écogéographie de J. Tricart et J. Kilian (1979). D'après lui, ce courant s'est construit à compter du moment où "les géographes en sont venus aussi à s'intéresser sérieusement au problème de l'environnement; dans le domaine de la géographie physique, on observe que beaucoup d'entre eux ont évolué en passant de travaux spécifiques sur la morphologie, le climat, l'hydrologie, etc. à des recherches plus amples sur l'environnement ou en poursuivant leurs travaux dans des domaines spécifiques, ils ont commencé à appliquer les connaissances spécialisées, en prenant en compte de l'impact des éléments naturels sur l'environnement, lorsque ceux-ci sont influencés par la société [...] ».

31 D’après M. C. Andrade (1987), au Brésil, le développement d'une approche en accord avec la perspective d'une géographie écologique serait initialement dû à Hilgard O'Railly Sternberg, Aziz Ab'Saber et Carlos Augusto de Figeiredo Monteiro, à la fin des années 1960 et au début des années 1970. Prenant en considération les apports de ce courant pour l'évolution de la pensée géographique, l'auteur cité ci-devant semble expliciter des modifications de son point de vue en montrant, dans des ouvrages plus récents (Andrade, 1994, 1999), la participation de géographes plus sensibles aux sciences humaines, dans l'approche de l'environnement. D'après lui : « De nos jours, grâce à la reconnaissance des problèmes environnementaux, soit dus à l'exploitation désordonnée des ressources, pas toujours renouvelables, soit à la pollution de l'eau et de l'atmosphère, les études environnementales bénéficient d'une approbation de plus en plus grande et se distribuent tant en travaux exclusivement géographiques qu'en travaux interdisciplinaires » (1999).

On observe ainsi une progression considérable de l'approche des problèmes environnementaux dans les années 1980 et 1990, c'est-à-dire, d'une phase caractérisée surtout par l'approche écologique, dans laquelle on mettait en valeur la tendance naturaliste, on est passé à une autre phase centrée sur l'environnement, dans laquelle, société et nature composent les deux parties d'une interaction dialectique. Cette perspective géographique de l'approche environnementale n'est, toutefois, pas récente, puisqu'on peut l'identifier dans les idées d'élisée Reclus exprimées il y a près de cent ans et qui, malgré une solution de continuité de plus d'un demi-siècle dans sa diffusion et application, ont été reprises et améliorées aujourd'hui. Dans ce courant, la problématique environnementale dans la géographie n'est plus identifiée comme uniquement liée à la géographie physique, mais devient géographique à part entière. Cette phase actuelle du développement de la pensée géographique semble répondre à l'appel suivant de $\mathrm{C}$. A. F. Monteiro (1984), un des principaux précurseurs du courant socio-environnemental de la géographie, lancé il y a près de 20 ans.

Un entendement philosophique favorable à l'approche scientifique de la problématique environnementale actuelle peut tout aussi bien être trouvé dans diverses propositions philosophiques produites depuis la Grèce Classique que, dans les limites de ces dernières, exiger de nouvelles formulations comme le postulent, entre autres, F. Capra (1987), M. Serres (1988), E. Morin et A. B. Kern (1995), et H. Leff (2001). Dans le cadre de la géographie, les études liées à cette problématique ont, d'un côté, été développées à partir des nuances philosophiques les plus diverses, normalement utilisées par cette science, 
donnant lieu alors à des extrémismes singuliers qui exacerbent l'approche en faveur du naturel ou du social, et d'un autre, elles ont exigé des ruptures de la configuration actuelle de la production géographique. De telles ruptures ont vu le jour tant sur le plan théorique que méthodologique et technique de la recherche de la dimension spatiale de la susdite problématique puisque, comme l'a reconnu A. C. R. Moraes (1994) en introduisant une discussion relative à l'épistémologie pour étudier l'environnement, il s'agit d'une «[...] nouvelle question, [...] qui refuse les modèles traditionnels $[. . .]^{1}{ }^{1}$.

Si dans la société en général, le terme environnement (environment, environnement), victime de l'inflation journalistique, est devenu banal, comme le montrent A. Bailly et $R$. Ferras (1997), dans le cadre académico-scientifique, il a aussi souffert de ce processus. De tels termes sont utilisés de la manière la plus diverse mais, le plus souvent, ils sont employés à l'instar des procédés industriels qui collent de nouvelles étiquettes sur des produits hors circuit ou qui ne correspondent pas au contenu exprimé. Il faut alors faire très attention, car les étiquettes peuvent rendre les produits plus attractifs et exprimer leur caractère avant-gardiste, mais elles peuvent aussi être très trompeuses.

Il faut donc être très prudent et attentif, car tout ce qui est géographique n'est pas forcément environnemental. Dans ce sens, il faut aussi signaler que tout ce qui est produit dans la perspective de la géographie physique ne doit pas recevoir l'étiquette d'environnemental, parce que beaucoup de travaux n'en n'ont pas la forme, même s'ils contribuent de façon importante à ces derniers. La caractéristique environnementale d'une étude ne fait pas que celle-ci soit meilleure ou pire qu'une autre élaborée selon d'autres courants, qui relèvent de la géographie ou d'une autre science; elle en fait une étude différente des autres tout simplement.

Dans l'acception défendue ici, une étude élaborée dans la voie d'une géographie socioenvironnementale doit venir de problématiques où des situations conflictuelles, conséquences de l'interaction entre la société et la nature, montrent clairement la détérioration de l'une ou des deux. C'est la diversité des problématiques qui va exiger une approche plus centrée sur la dimension naturelle ou sociale, en réponse au fait que l'objectif principal de telles études et actions va dans le sens d'une recherche de solutions du problème, et que celui-ci devra être abordé à partir de l'interaction entre ces deux composants de la réalité

D'un autre côté, il faut aussi insister sur ce point, toute production qui procède de la géographie peut être utile à l'approche d'études environnementales, car en fin de compte, une des dimensions les plus importantes de la problématique environnementale correspond à la manifestation spatiale de cette dernière. La géographie écologique et la géographie environnementale représentent, ainsi, deux spécialités d'un même courant, ici considéré comme géographie socio-environnementale: la première revêt une caractéristique naturaliste plus marquée, tandis que la seconde valorise une approche qui envisage la nature et la société dans une perspective unique, choisissant l'angle socioenvironnemental pour mettre en valeur ce point de vue, comme il a été dit antérieurement. 


\title{
La géographie socio-environnementale et la question méthodologique : en rupture avec les paradigmes de la science moderne
}

\author{
« La crise environnementale est la crise de notre \\ temps. Le risque écologique remet en question la \\ connaissance du monde. Cette crise se présente à \\ nous comme une limite dans le réel, qui re-signifie \\ et réoriente le cours de l'histoire : limite de \\ croissance économique et de population ; limite \\ des déséquilibres écologiques et des capacités de \\ durabilité de vie ; limite de la pauvreté et de \\ l'inégalité sociale. Mais aussi crise de la pensée \\ occidentale [...]. » \\ (Leff, 2001)
}

Une des principales caractéristiques de la science moderne - à partir de la phase la plus évoluée de la modernité (d'après $M$. Berman, 1986) - correspond à l'emploi de méthodes de recherche dans la production du savoir scientifique. Les postulats positivistes qui conditionnent la structure de la science confinent encore - même aujourd'hui - la lecture de la réalité à des filières disciplinaires, réduisant ainsi l'appréhension de celle-ci à des perspectives séparatrices, étanches et appauvrissantes. L'approche de la problématique environnementale, dans un tel contexte, laisse toujours à désirer.

L'évolution de la géographie dans les 150 dernières années reflète directement les postulats positivistes qui imposent l'adoption de méthodes spécifiques à divers courants de pensée. De cette manière, le positivisme a été associé à la géographie classique (ou traditionnelle), le néo-positivisme à la New geography, l'humanisme à la géographie de la culture et de la perception, le marxisme à la géographie critique, etc.

\section{À quelle méthode est associée la géographie socio- environnementale?}

d'abord, cette question tend vers le constat qu'» [...] il n'existe pas seulement une méthode dans la science et il est urgent de comprendre les possibilités offertes par chacune d'elles dans l'équation de la thématique environnementale [...]» (Moraes, 1994), surtout face aux caractéristiques de ce courant, qui est multi et interdisciplinaire.

L'approche de la problématique environnementale, pour être menée à bien, en profondeur et dans le sens de l'interaction société-environnement, rompt ainsi avec un des postulats classiques de la science moderne, en l'occurrence celui qui établit le choix d'une seule et unique méthode pour élaborer le savoir scientifique. Cette approche exige tant l'application de méthodes déjà expérimentées dans le champ de diverses sciences spécifiques, que la formulation de méthodes nouvelles. Mais cette caractéristique n'est pas propre à la seule approche environnementale, elle reflète la véritable identité de la géographie dans nombre de ses expériences, car comme le dit F. Trystram (1994) : «[...] Interface, le mot choisi par Philippe Pinchemel est révélateur. La géographie n'est ni une touche à tout ni une bonne à rien. Elle est à la croisée des nombreux chemins qui mènent 
de l'anthropologie à la sociologie, de la nature à l'écologie, des sciences de la Terre aux statistiques [...].»

L'abandon du positivisme dans la géographie n'est pourtant pas un désir très récent. Nombreuses furent les propositions qui ont cherché à faire interagir des méthodes de branches appartenant à la géographie ou à différentes disciplines dans une même étude. En ce qui concerne l'étude de l'environnement, on remarque les perspectives de la production d'une géographie physique globale basée sur l'interaction de méthodes qui prennent la perspective verticale (écosystème) et horizontale (géosystème) des paysages, prenant en compte aussi les activités humaines en tant que facteurs de la dynamique du paysage. On souligne dans l'histoire récente de la géographie les contributions de Sotchava (géosystème) - améliorée par Georges Bertrand -, et celles de J. Tricart (écodynamique et écogéographie), entre autres (Mendonça, 1989, 1993 et 1998a; Christofolleti, 1999).

Les propositions méthodologiques citées se basent sur la TGS (Théorie Générale des Systèmes), très utilisée dans les sciences naturelles, qui a lié l'approche de l'environnement au sein de la géographie - par le biais de méthodologies diverses - à une perspective beaucoup plus naturaliste que sociale. Ceci s'explique, comme l'écrit K. J. Grégory (1992), du fait que «le danger d'adopter une perspective systémique sans la critiquer consiste dans le fait que l'on présume suffisant de se limiter à identifier les structures du système et de décrire les nombreuses variables concernées dans un système particulier ; système qui renforce alors la première loi de l'Écologie, comme elle a été graphiquement annoncée par Commoner (1972), selon laquelle tout est relié à tout [...] ».

Mais, bien que partiales et limitées, et durement critiquées par B. M. Soares Pontes (1999), il faut attribuer un grand mérite aux méthodologies citées ci-dessus à perspective globalisante au sein de la géographie physique, car elles ont essayé de faire interagir société et nature dans une même approche, et d'une certaine manière, elles y ont réussi entre autres exemples dignes d'être cités, voir le travail de C. A. F. Monteiro (1987) à propos du Recôncavo Baian'2. Ces méthodologies ont contribué tant à l'amélioration de la géographie physique qu'à la construction de la géographie socio-environnementale dont il est question ici.

45 Bien que, depuis les années 1950, il soit possible d'observer la marque d'une certaine préoccupation pour l'approche globale au sein de la géographie physique, qui lui a valu le caractère d'environnementale - quoique partiale, l'argument suivant de R. H. Briant (cité par Derruau, 1996) complète les affirmations présentées ici selon lesquelles «[...] Il ne suffit pas, pour être clair, de définir la géographie physique comme étant "l'étude intégrée du milieu (environnement) naturel à la superficie ou à la proximité immédiate de la superficie de la terre" ", car pour plus ouverte qu'elle soit, elle considère encore l'homme et la société comme facteurs et non comme éléments du paysage.

Lorsqu'il situe l'origine et le développement de la géographie écologique comme liée à la géographie physique, M. C. Andrade (1987) reconnaît l'ouverture que ces géographes ont connu en se lançant dans une nouvelle conception et production de l'étude de l'environnement du point de vue géographique, car, à son avis, « sur beaucoup de points, ils [les spécialistes en géographie physique ${ }^{3}$ se rapprochent du groupe des dits géographes critiques ou radicaux, alors que sur d'autres, ils entrent en contact avec le groupe des géographes de la perception et du comportement». Cette constatation reflète, entre autres, les limites des méthodes disciplinaires individuellement inhérentes à la science moderne pour traiter la réalité et les problèmes environnementaux. Elle révèle, encore, la 
nécessité d'appliquer une perspective multi et interdisciplinaire au sein de la géographie et d'appliquer cette dernière à d'autres sciences, car «[...] la discussion à propos de la question environnementale devra transiter à l'intérieur de cadres disciplinaires [...]» (Moraes, 1994).

Tout en se rapprochant des géographes critiques, comme l'a affirmé M. C. Andrade (1987), les géographes physiciens n'ont pas réussi à insérer le marxisme en tant que méthodologie centrale de leur analyse, ni dans les études physico-géographiques, ni dans les études socio-environnementales qu'ils ont élaborées, car « une approche marxiste de la question environnementale va l'envisager comme une manifestation de processus sociaux, pour lesquels une société donnée organise l'accès et l'usage des ressources naturelles disponibles, organisation qui s'articule dans la propre structuration sociale qui fait partie du processus global de sa reproduction [...]» (Moraes, 1994).

Bien que la perspective marxiste soit un instrument d'une valeur inestimable dans l'analyse de la dynamique sociale - en particulier à compter du matérialisme historique et dialectique -, ou même que d'autres méthodes comme le structuralisme, le fonctionnaliste, etc., contribuent beaucoup à l'entendement de la société, l'approche environnementale ne sera toujours élaborée que partiellement si elle est focalisée sur une seule d'entre elles, parce que le social ne compose qu'une seule parcelle de l'environnement. Les éléments de la nature ne doivent pas être réduits aux seules ressources, car avant d'être ainsi transformés, ils se constituent en biens et en éléments naturels qui possèdent une dynamique propre, indépendante de l'appropriation sociale qui en est faite ; à ce titre, ils jouent un rôle fondamental dans la structuration de l'espace géographique. Même s'ils font partie d'espaces dominés par l'homme et sa société, ils n'échappent pas au contrôle du flux de matière ni d'énergie qui régie l'existence du système solaire, de la planète Terre et de ses composants. Il est vrai qu'en nombreux endroits - comme les grandes villes, par exemple -, on a la fausse impression que l'homme est un grand chef d'orchestre, que «la nature » et ses forces ou ressources n'existent plus ou bien ont été subjuguées aux desseins humains. Il est même incroyable que, dans une approche géographique, soient oubliés le relief qui est à la base de l'existence de la ville, l'eau et l'air indispensables à la vie de ceux qui l'habitent, les aliments produits par le sol qui les nourrit, etc.

En focalisant la tendance environnementale de la géographie et la nécessaire ouverture méthodologique que celle-ci contient, C. A. F. Monteiro (1980) montrait clairement, à la fin des années 1970 et justement au moment où la géographie brésilienne devenait très marquée par la géographie critique, qu'elle est constituée «[...] de géographes qui, tout en considérant le prisme anthropocentrique de la géographie, l'identifient principalement à la science des lieux. Tout en prenant en compte l'augmentation progressive du pouvoir de décision et la capacité de changement anthropique, les "Systèmes" organisés à la surface de la Terre, se comporteront toujours comme des systèmes naturels et, à ce titre, ils doivent êtres étudiés. Ceci sous l'impact idéologique universel de la "croisade en faveur de l'environnement", sous le slogan "nous n'avons qu'une seule terre" [...]» (Monteiro, 1980).

Il montrait aussi, et avec justesse, que le courant environnemental ne devrait pas être considéré comme prédominant dans la géographie, mais seulement comme une possibilité supplémentaire offerte à la réflexion des géographes sur le monde, puisque "les vecteurs, qui conduisent à la notion de géographie en tant que science sociale, seront prédominants [...]». À son avis, «l' environnementalisme » dans la géographie 
n'effacerait pas «[...] le développement ou la poursuite de lignes centrales de pensée [...], ni, très probablement, n'empêcher[ait] les progrès sectoriels les plus extrêmes avant qu'ils ne réussissent à dépasser les limites de démarcation de la recherche " géographique » pour se développer de manière autonome [...] » (Monteiro, 1980).

L'objet d'étude de la géographie socio-environnementale, œuvre contemporaine de l'interaction entre la nature et la société, ne peut être conçu comme dérivant d'une réalité dont les deux composants se comportent de façon étanche et indépendante, puisque c'est la relation dialectique de ces derniers qui constitue sa propre base.

Le courant de la géographie socio-environnementale, est ancré dans la conception selon laquelle «le point épistémologique le plus important pour la géographie se trouve dans l'attitude phénoménologique de ne considérer ni la Nature (matière de l'expérience), ni l'Homme (corps perceptif) comme "fondateurs" [fundantes] ( (Monteiro, 1984). Il s'agit, sans aucun doute, d'une identification innovatrice dans et de la géographie environnementale -, originale, mais qui n'exclut aucune des autres possibilités de développement du savoir géographique.

Reprenant Merleau-Ponty, Monteiro poursuit en montrant qu'on peut identifier les traits les plus généraux, mais non exclusifs, de la perspective méthodologique de ce courant de la géographie contemporaine, ici nommée courant de la géographie socioenvironnementale : «L'homme et la société ne se trouvent pas exactement en dehors de la nature et du biologique - ils s'en distinguent par le fait qu'ils concentrent en eux les " paris » de la nature, en les faisant tous à la fois. C'est pourquoi l'“environnemental" ne doit pas être seulement envisagé au niveau biologique ou écologique, mais surtout par ce qu'il contient de construction holistique.»

De part sa nature multi et interdisciplinaire et sa perspective holistique dans la conception de l'interaction établie entre la société et la nature, le courant de la géographie socio-environnementale prend la forme d'un champ utile à l'exercice de l'éclectisme méthodologique, car «en tant qu'abstraction humaine de la réalité, les méthodes et les techniques doivent être considérées comme n'appartenant à aucun savoir en particulier, mais momentanément requises pour une science ou une autre » (F. Joly, dans Mendonça, 1998). Éclectisme n'est pas, signalons-le, synonyme de pot-pourri, autrement dit, il ne signifie pas de faire « du n'importe quoi »; la logique, le sérieux, et la cohérence dans le choix de méthodologies et de techniques qui s'adaptent bien à la problématique d'étude sont des qualités nécessaires pour obtenir des résultats concis dans des études à caractère socio-environnemental.

\section{Conclusion}

En identifiant le courant de la pensée géographique géographie socio-environnementale à partir de la délimitation de sa construction historique, même si elle est généralement présentée ici comme une introduction et une contribution à la discussion de l'épistémologie de la géographie contemporaine, on découvre d'autres arguments en faveur de la constatation selon laquelle la géographie est vraiment un «savoir difficile", comme le montrent $\mathrm{P}$. et $\mathrm{G}$ Pinchemel (épigraphe de ce texte). En identifiant ce courant comme un champ d'analyse parmi d'autres pour le géographe, on ne défend pas sa supériorité par rapport aux autres, car la richesse de la pensée géographique repose sur la pluralité de ses approches. 

mais nous pensons avoir montré qu'une partie de ces dernières permet de le distinguer dans le panorama de la science géographique contemporaine. La nature changeante du monde contemporain et l'accélération de ses changements, imposent l'application nécessairement simultanée de nouvelles vues, de nouvelles techniques et de nouvelles perspectives à l'objet d'étude de la géographie. Elle impose surtout l'ouverture des esprits en vue de créer de la nouveauté, de la différence, capable de dépasser le niveau de difficultés et de limites de l'appréhension du réel qui caractérise, très fortement encore, le présent. Une nouvelle pensée, à l'origine de changement, ne se consolide pas sans un dialogue entre les différents savoirs ni sans dissuader les opposants, et ces derniers finissent par la parfaire en lui permettant d'oser et de repenser en profondeur ses formulations. Si elle se montre incapable d'assimiler les apports de ce processus et de mûrir plus vite que prévu, il est préférable alors qu'elle ait une vie courte ou qu'elle ne soit même pas mise en œuvre.

Toutefois, la crise environnementale contemporaine - crise de civilisation, crise de raison et crise historique - comme nous l'avons montré tout au long de ce texte, exige de toute la société, et de la science en particulier, une réflexion profonde sur l'orientation qu'elle prend. Dans un tel contexte, il faut être suffisamment ouvert, créatif et osé pour proposer des changements et créer les possibilités pour que naissent de nouvelles propositions, comme la géographie socio-environnementale ici présentée.

pas nécessaire d'être partisan de radicalismes environnementaux ou écologiques pour comprendre l'importance des éléments de la nature dans la constitution de la société humaine, surtout face aux excès de dégradation de celle-ci par la société qui se l'approprie, obéissant au projet de la modernité, comme l'a si bien signalé M. Serres (1988, 1994) - pourtant personnellement partisan de l'Écologie Radicale. Un rôle fondamental s'impose, alors, à la géographie dans la construction d'un monde nouveau, d'une vie nouvelle. $\mathrm{Du}$ point de vue de la problématique environnementale contemporaine, sa contribution sera beaucoup plus efficace et profonde si elle est élaborée en accord avec le courant de la géographie socio-environnementale, ici ébauchée.

\section{BIBLIOGRAPHIE}

AMARAL PEREIRA (R. M. P.), 1989. - Da geografia que se ensina à geografia moderna, Florianópolis, UFSC. ANDRADE (M. C.), 1987. - Geografia - Ciência da sociedade : Uma introdução à analise do pensamento geográfico, São Paulo, Atlas.

-, 1994. - O desafio ambiental, São Paulo, HUCITEC.

-, 1999. - « Aconstrução da geografia brasileira », RA'E GA - O espaço geográfico em analise, $\mathrm{n}^{\circ}$ 3, III, p. 19-34. 
BAILLY (A.), FERRAS (R.), 1997. - Éléments d'épistémologie de la géographie, Paris, Armand Colin, coll.

«U Géographie », 191 p.

BERMAN (M.), 1986. - Tudo que é solido desmancha no ar : A aventura da modernidade, São Paulo, Companhia das Letras.

BRAVARD (J.-P.), 1999. - « Pourquoi les géographes phyciens gémissent-ils? », Annales de Géographie , nº 606, p. 198-200.

CAPRA (F.), 1987. - O ponto de mutação - A ciência, a sociedade e a cultura emergente, São Paulo, Cultrix. CHRISTOFOLETTI (A.) et al., 1999. - Modelagem de sistemas ambientais, São Paulo, Edgard Blucher.

CLAVAL (P.), 2001. - « A revolução pós-funcionalista e as concepções atuais da geografia », dans Mendonça (F. A.), Khozel (S.) (dir.), Elementos de epistemologia da Geografia contemporânea, Curitiba, Editora da UFPR.

DERRUAU (M.), 1996. - Composantes et concepts de la géographie physique, Paris, Armand Colin, coll. «U Géographie », 256 p.

FOLLADORI (G.), 1999. - Los límites del desarrollo sustentable, Montevidéu, Banda Oriental.

GREGORY (K. J.), 1992. - A natureza da geografia física, Rio de Janeiro, Bertrand Brasil.

hoBsBawm (E.), 1995. - A era dos extremos, São Paulo, Ática.

LEFF (H.), 2001. - Epistemologia ambiental, São Paulo, Cortez.

MASSEY (D.), 1999. - « Space-time, "science" and the relationship between physical geography and human geography », Royal Geographical Society, n²4, p. 261-276.

MENDONÇA (F. A.), 1989. - Geografia física : Ciência humana ?, São Paulo, Contexto, $1^{\text {re }}$ édition.

-, 1993. - Geografia e meio ambiente, São Paulo, Contexto, $1^{\text {re }}$ édition.

-, 1998. - « Dualidade e dicotomia da geografia moderna : A especificidade científica e o debate recente no âmbito da geografia brasileira ", RA'E GA - O espaço geográfico em análise, $\mathrm{n}^{\circ} 2$, II, $\mathrm{p}$. 153-166.

-, 1998a. - « Geografia e metodologia científica - Da problemática geral às especificidades da geografia física », Geosul, vol. 14, n² 27, p. 63-70.

Monteiro (C. A. F.), 1980. A geografia no Brasil (1934-1977) - Avaliação e tendências, São Paulo, IGEO/ USP.

-, 1981. - A questão ambiental no Brasil : 1960-1980, São Paulo, IGEO/USP.

-, 1984. - « Geografia \& ambiente », Orientação, n 5, p. 19-28.

-, 1987. - Qualidade ambiental na Bahia - Recôncavo e regiões limítrofes, Salvador, Governo da Bahia/ SEPLANTEC/Centro de Estatística e Informações.

-, 1988. - « Travessia da crise (tendências atuais na geografia) », dans Revista Brasileira de Geografia, Rio de Janeiro, $50^{\mathrm{e}}$ année, numéro spécial, t. 2, p. 127-150.

-, 1999. - « A abordagem ambiental na geografia - possibilidades na pesquisa e limitações do geógrafo ao monitoramento », RA'E GA - O espaço geográfico em análise, n 3, III, p. 9-18.

MORAES (A. C. R.), 1994. - Meio ambiente e ciências humanas, São Paulo, Hucitec.

MORIN (E.), Kern (A. B.), 1995. - Terra Pátria, Porto Alegre, Sulina.

PORTO GONÇALVES (C. W.), 1989. - Os (des)caminhos do meio ambiente, São Paulo, Contexto. 
SERRES (M.), 1988. - Le contrat naturel, Paris, Flammarion, coll. « Champs ; 241 », 192 p.

-, 1994. - La philosophie et le climat, Conférence du Colloque de Lassay/França.

SOARES PONTES (B. M.), 1999. - « A ciência geográfica e o desafio da questão natureza/sociedade », Sociedade \& natureza, $11^{\mathrm{e}}$ année, $\mathrm{n}^{\circ} 21-22$, p. 29-44.

TRICART (J.), KILLIAN (J.), 1979. - « L'éco-geographie et l'aménagement du milieu naturel », Herodote , numéro spécial.

TRYSTRAM (F.), 1994. - Terre! Terre! De l'Olympe à la Nasa, une histoire des géographes et de la géographie, Paris, Lattès, coll. « Les traversées de l'histoire », 494 p.

VeYret (Y.), 1999. - Géo-environnement, Paris, Armand Colin, coll. « Campus géographie », 186 p.

WHITEJR (L.), 1968. - Machina ex Deo : Essays in the dynamism of western culture, Cambridge (Mass.), The MIT press.

\section{NOTES}

1. Souligné par Moraes lui-même.

2. Note de la traductrice : ce terme se réfère à la région étendue et fertile de Bahia.

3. Expression de M. C. Andrade dans une autre partie du même paragraphe.

\section{RÉSUMÉS}

L'environnement est un des éléments constituant de la science géographique moderne. Il met en évidence la richesse dualiste de la connaissance géographique. La conception de l'environnement, toutefois, a beaucoup changé au cours du siècle dernier car elle a subi différentes influences. Dès le milieu $\mathrm{du} \mathrm{xx}^{\mathrm{e}}$ siècle la géographie, les autres sciences et même la société en général, concevaient l'environnement exclusivement à partir d'une perspective naturaliste. Dans les quarante dernières années la notion de l'environnement a intégré, progressivement, la dimension sociale, car la crise contemporaine de l'environnement ne peut pas être conçue et résolue non plus par les approches traitant séparément la nature et la société. Dans ce contexte, la problématique environnementale actuelle demande à la géographie de revoir ses conceptions en tenant compte des nouvelles propositions théoriques et méthodologiques des sciences de l'environnement. La prise en compte de la société et de la nature dans les études liées à la problématique environnementale, dans laquelle nature et société sont conçues comme éléments du processus, donne naissance à un nouveau courant de la connaissance géographique nommée dans ce texte de géographie socio-environnementale.

The geography, since it's established as a modern science, has in the environmentalism one of its principal characteristics. It reflects the richness of duality of the geographical knowledge. The conception of environment, however, changed a lot during the last century, because it's inserted very important frames from different historical moments of modernity. Since the twenties middle the geography, and also the other sciences and society in general, conceived the environment exclusively on the natural perspective. On the last forty years the notion of 
environment has incorporated, progressively, the social dimension, because the contemporary environmental crisis can't be understood and neither resolved by the perspectives that separates society and nature. In this context, the environmental problematic which characterizes the present moment leads the geography to revise its conceptions, and it resulted on the search and on the formulation of new theory-methodology bases to the environment approach. The involvement of society and nature in the studies emanated from environmental problematic, in which the natural and social are conceived as elements belonged to a same process, resulted in a new current of geographical knowledge here called socio-environmental geography.

INDEX

Mots-clés : environnement, géographie, géographie écologique, géographie socioenvironnementale

Keywords : ecological geography, environment, geography, socio-environmental geography Index géographique : Brésil

\section{AUTEUR}

\section{FRANCISCO MENDONÇA}

Département de Géographie, Université Fédérale du Paraná, Centro Politecnico, Jardim das Americas, 81531-970 - Curitiba, Brésil,

chico@ufpr.br 